\title{
INTERFERENCE SHAPING CONSTRAINTS FOR UNDERLAY MIMO INTERFERENCE CHANNELS
}

\author{
C. Lameiro, I. Santamaría*
}

\author{
Dept. of Communications Engineering \\ University of Cantabria, Spain \\ \{lameiro,nacho\}@gtas.dicom.unican.es
}

\author{
W. Utschick ${ }^{\dagger}$
}

\author{
Associate Institute for Signal Processing \\ Technische Universität München, Germany \\ utschick@tum.de
}

\begin{abstract}
In this paper, a cognitive radio (CR) scenario comprised of a secondary interference channel (IC) and a primary point-to-point link (PPL) is studied, when the former interferes the latter. In order to satisfy a given rate requirement at the PPL, typical approaches impose an interference temperature constraint (IT). When the PPL transmits multiple streams, however, the spatial structure of the interference comes into play. In such cases, we show that spatial interference shaping constraints can provide higher sum-rate performance to the IC while ensuring the required rate at the PPL. Then, we extend the interference leakage minimization algorithm (MinIL) to incorporate such constraints. An additional power control step is included in the optimization procedure to improve the sum-rate when the interference alignment (IA) problem becomes infeasible due to the additional constraint. Numerical examples are provided to illustrate the effectiveness of the spatial shaping constraint in comparison to IT when the PPL transmits multiple data streams.
\end{abstract}

Index Terms - Cognitive radio, interference alignment, interference channel, interference temperature

\section{INTRODUCTION}

Typically, when two or more networks coexist over the same radio resources, orthogonal resource allocation approaches are applied to eliminate inter-network interference, which is clearly suboptimal. In this context, cognitive radio (CR) has been proposed as a promising approach to efficiently utilize the scarce radio resources $[1,2]$. In underlay CR systems, the secondary users are allowed to coexist with a primary link over the same resources, as long as the interference level generated at the former is such that a given rate is guaranteed. To this end, the secondary users are usually constrained by the socalled interference temperature (IT), so that the interference level at the primary receiver is limited to a maximum value.

In this paper, we consider a coexistence scenario in which a point-to-point link (PPL) is willing to share its spectrum with an interference channel (IC), provided that the PPL rate requirements are satisfied. Outside the context of $\mathrm{CR}$, the $K$-user multiple-input multiple-output (MIMO) IC is currently receiving a great deal of attention. In such channels, $K$ transmitter communicate with their corresponding receivers generating interference to the unintended ones.

${ }^{*}$ The research leading to these results has received funding from the Spanish Government (MICINN) under projects TEC2010-19545-C04-03 (COSIMA), CONSOLIDER-INGENIO 2010 CSD2008-00010 (COMONSENS), and FPU Grant AP2010-2189.

${ }^{\dagger}$ This research has been funded by the Deutsche Forschungsgemeinschaft (DFG) under the grant Ut36/15-1.
Interference alignment (IA) has emerged as an interesting approach to manage interference in such scenarios, allowing each user to communicate free of interference and to approach capacity in the asymptotic signal-to-noise ratio (SNR) regime [3]-[5]. Although there are still many open problems regarding this technique, different studies carried out over the last years have provided many interesting theoretical results [6]-[9], as well as many different algorithms to perform IA [10]-[17]. Among those, it is worth highlighting the interference leakage minimization algorithm (MinIL) proposed in [10].

$\mathrm{CR}$ with $K$-user IC as secondary network has been studied in [18]-[22]. In [18], the achievable degrees-of-freedom (DoF) are studied when the primary user performs the optimal strategy, namely, singular value decomposition (SVD) and waterfilling power allocation, which may result in some unused eigenmodes, in which the IC opportunistically confines the transmitted signals. On the other hand, the authors in [19] and [20] follow the same idea but show that, if the primary receiver cooperates and performs interference suppression decoding, the sum-rate of the IC is significantly improved with negligible primary rate reduction. A non-iterative IA scheme is proposed in [21], in which the IC is also constrained to cause zero interference to the primary. In [22] a slightly modified scenario is studied, in which the IC interferes the primary user but not the other way around, and some level of interference is allowed. Taking this into account, the authors of [22] propose an IA algorithm that minimizes the interference leakage (IL) subject to IT constraint to control the interference level at the primary, based on semidefinite relaxation (SDP) and randomization techniques. Coexistence issues have been also considered for other networks. For instance, in [23], the performance degradation due to IT is analyzed for a secondary user that coexists with multiple primary users. In [24] and [25], the transmission strategy of a secondary user that shared the spectrum with a primary is optimized subject to an explicit rate constraint on the former, which requires additional channel state information (CSI) on the secondary transmitter. Also, single-input single-output (SISO) or multiple access (MAC) secondary networks are considered in [26] and [27], respectively.

In this paper, we consider the same model as in [22]. We first show that, when the primary link transmits multiple streams, constraining the spatial structure of interference [28] is crucial for the IC to achieve good sum-rate performance. To this end, we derive transmit shaping constraints at the secondary transmitters and extend the MinIL algorithm to incorporate such constraints. An additional power control step is introduced to enhance the sum-rate of the IC when the data rate requirement of the primary link is high. With the proposed approach, the IC requires no CSI from the primary and needs only to know the aforementioned constraints to design the transmit directions. 


\section{SYSTEM MODEL}

We consider a $K$-user single-beam MIMO IC that coexists with a MIMO PPL. We assume that the PPL transmitter is deployed far from the IC, and therefore the latter receive no interference from the former. Under this setting, and denoting by $M_{i}$ and $N_{j}$ the number of antennas at receiver $i$ and transmitter $j$, respectively $(i, j=$ $0, \ldots, K$, where $i, j=0$ denotes the PPL), the signal received by each user can be expressed as

$$
\begin{aligned}
& y_{i}=\mathbf{u}_{i}^{H}\left(\sum_{j=1}^{K} \mathbf{H}_{i j} \mathbf{v}_{j} \mathbf{s}_{j}+\mathbf{n}_{i}\right), i=1, \ldots, K \\
& y_{0}=\mathbf{U}_{0}^{H}\left(\mathbf{H}_{00} \mathbf{V}_{0} \mathbf{s}_{0}+\sum_{j=1}^{K} \mathbf{H}_{0 j} \mathbf{v}_{j} s_{j}+\mathbf{n}_{0}\right),
\end{aligned}
$$

where $\mathbf{H}_{i j} \in \mathcal{C}^{N_{i} \times M_{j}}$ is the MIMO channel between transmitter $j$ and receiver $i, \mathbf{U}_{0} \in \mathcal{C}^{N_{0} \times N_{0}}$ and $\mathbf{V}_{0} \in \mathcal{C}^{M_{0} \times M_{0}}$ are the decoding and precoding matrices of the PPL, respectively; $\mathbf{u}_{i} \in \mathcal{C}^{N_{i} \times 1}$ and $\mathbf{v}_{i} \in \mathcal{C}^{M_{i} \times 1}$ are the decoding and precoding vectors of the $i$ th IC user, respectively; $\mathbf{n}_{i} \in \mathcal{C}^{N_{i} \times 1}$ is the noise at receiver $i$ which is assumed to be distributed as $\mathcal{C N}\left(0, \sigma^{2} \mathbf{I}\right)$ and $\mathbf{s}_{0} \in \mathcal{C}^{M_{0} \times 1}$ and $s_{j} \in$ $\mathcal{C}$ are the symbols transmitted by the PPL and the $j$ th IC transmitter, respectively, which are distributed as $\mathcal{C N}(0, \mathbf{I})$.

Let us assume that the PPL does not know the actual interference covariance matrix and performs the optimal transmission strategy, namely, SVD and waterfilling power allocation [29]. Thus, its achievable rate as a function of the aggregate interference covariance matrix, $\mathbf{Q}=\sum_{j=1}^{K} \mathbf{U}_{0}^{H} \mathbf{H}_{0 j} \mathbf{v}_{j} \mathbf{v}_{j}^{H} \mathbf{H}_{0 j}^{H} \mathbf{U}_{0}$, is given by

$$
R_{\mathrm{PPL}}(\mathbf{Q})=\log \operatorname{det}\left(\mathbf{I}+\left(\sigma^{2} \mathbf{I}+\mathbf{Q}\right)^{-1} \boldsymbol{\Sigma}_{S}\right),
$$

where $\boldsymbol{\Sigma}_{S}$ is a diagonal matrix. The minimum rate constraint can be therefore expressed as $R_{\mathrm{PPL}}(\mathbf{Q}) \geq(1-\alpha) R_{\mathrm{PPL}}(\mathbf{0})$, with $\alpha \in$ $[0,1]$.

An interesting issue that comes up at this point is related to the knowledge that each network has about the other one and how they cooperate with each other. In this work we consider that they have limited knowledge about each other and the required overhead is reduced as much as possible. More specifically, each transmitter of the IC only needs to know its transmit covariance constraint in order to optimize its precoders and decoders. On the other hand, the PPL only needs to know the local channels, $\mathbf{H}_{0 i}$, in order to select suitable constraints.

\section{INTERFERENCE SHAPING CONSTRAINTS}

In this section we derive shaping constraints [28] for the IC to ensure that the rate requirement at the PPL is satisfied. We assume that the constraints are obtained by the PPL receiver, which has perfect local CSI, and are sent to the IC through a feedback link. Typically, IT constraints are used to control the total interference power that the secondary users generate at the primary receiver. When the PPL uses single-beam transmissions there is not much left to do; however, in the multiple-stream case, how the interference is distributed among the different streams strongly affects the achievable rate of the PPL. To this end, we will constrain the spatial interference distribution by using the following matrix partial orders on the set of $N \times N$ positive semidefinite matrices

$$
\begin{array}{ll}
\mathbf{A} \preceq \mathbf{B} & \equiv \mathbf{B}-\mathbf{A} \in \mathcal{S}_{+}^{N}, \\
\mathbf{A} \preceq_{\mathcal{D}} \mathbf{B} & \equiv(\mathbf{A})_{i i} \leq(\mathbf{B})_{i i}, i=1, \ldots, N,
\end{array}
$$

where $\mathcal{S}_{+}^{N}$ is the set of $N \times N$ positive semidefinite matrices and $(\mathbf{A})_{i i}$ denotes the $i$ th element of the diagonal of $\mathbf{A}$. Notice that (4) is the Löwner ordering [30], whereas (5) is also a valid partial order since it is reflexive, antisymmetric and transitive [31]. For a given $\alpha$, we first consider a constraint in the aggregate interference covariance matrix, $\mathbf{Q}$, as

$$
\mathbf{Q} \preceq_{\mathcal{D}} \mathbf{C}_{\alpha},
$$

where $\mathbf{C}_{\alpha} \in \mathcal{S}_{+}^{N_{0}}$ is the interference shaping constraint, which is a diagonal matrix since expression

$$
\operatorname{det}\left(\mathbf{I}+\left(\sigma^{2} \mathbf{I}+\mathbf{C}_{\alpha}\right)^{-1} \boldsymbol{\Sigma}_{S}\right) \leq \prod_{i=1}^{N_{0}}\left[1+\left(\overline{\mathbf{C}}_{\alpha}^{-1}\right)_{i i}\left(\boldsymbol{\Sigma}_{S}\right)_{i i}\right]
$$

where $\overline{\mathbf{C}}_{\alpha}=\sigma^{2} \mathbf{I}+\mathbf{C}_{\alpha}$, holds with equality only when $\overline{\mathbf{C}}_{\alpha}$ is diagonal, which is due to Sylvester's determinant theorem and Hadamard's inequality. This interference shaping constraint is then translated into a transmit covariance constraint, so that the IC requires no additional CSI from the PPL, that is expressed as

$$
\mathbf{v}_{j} \mathbf{v}_{j}^{H} \preceq \mathbf{S}_{j}, j=1, \ldots, K,
$$

where $\mathbf{S}_{j} \in \mathcal{S}_{+}^{M_{j}}$ is the transmit covariance constraint of user $j$ and such that (6) is satisfied for all possible transmit directions, $\mathbf{v}_{j}$. The reasons for choosing the matrix partial order (5) instead of the standard ordering (4) for the interference shaping constraint in (6) are formalized in the following lemmas.

Lemma 1. Let $\mathcal{L}=\left\{\mathbf{Q} \in \mathcal{S}_{+}^{N_{0}} \quad: \quad \mathbf{Q} \preceq \mathbf{C}_{\alpha}\right\}$ and $\mathcal{D}=\{\mathbf{Q} \in$ $\left.\mathcal{S}_{+}^{N_{0}}: \mathbf{Q} \preceq_{\mathcal{D}} \mathbf{C}_{\alpha}\right\}$ for a given positive diagonal matrix $\mathbf{C}_{\alpha}$. Then $\mathcal{L} \subset \mathcal{D}$.

Proof. Suppose that $\mathbf{Q} \preceq \mathbf{C}_{\alpha}$ for a given $\mathbf{Q}$. Therefore, $\mathbf{a}^{H} \mathbf{Q a} \leq$ $\mathbf{a}^{H} \mathbf{C}_{\alpha} \mathbf{a}$, for all $\mathbf{a}$. Setting $\mathbf{a}$ an all-zero vector with a one in the $i$ th entry, it is clear that $\mathbf{Q} \preceq_{\mathcal{D}} \mathbf{C}_{\alpha}$ also holds, which proves that $\mathcal{L} \subseteq \mathcal{D}$. To prove that $\mathcal{L}$ is strictly a subset of $\mathcal{D}$, suppose that $\mathbf{Q}={ }_{\mathcal{D}} \mathbf{C}_{\alpha}$ for a given $\mathbf{Q}$, i.e., $(\mathbf{Q})_{i i}=\left(\mathbf{C}_{\alpha}\right)_{i i}$ for all $i$. As the eigenvalues of any Hermitian matrix majorize its diagonal [30], it turns out that $\mathbf{Q} \npreceq \mathbf{C}_{\alpha}$, which concludes the proof.

The following lemma establishes the monotonicity of (3) with respect to the ordering defined in (5).

Lemma 2. Let $\mathbf{Q} \in \mathcal{S}_{+}^{N_{0}}$ be any matrix such that $\mathbf{Q} \preceq_{\mathcal{D}} \mathbf{C}_{\alpha}$, for a given positive diagonal matrix $\mathbf{C}_{\alpha}$. Then $R_{P P L}(\mathbf{Q}) \geq R_{P P L}\left(\mathbf{C}_{\alpha}\right)$, where $R_{P P L}(\cdot)$ is given by (3).

Proof. To prove the lemma, we must show that the off-diagonal elements of $\mathbf{Q}$ do not reduce the achievable rate when its diagonal is fixed. To this end, let us consider that $\mathbf{Q}={ }_{\mathcal{D}} \mathbf{C}_{\alpha}$, i.e., $\mathbf{Q}=\mathbf{C}_{\alpha}+\boldsymbol{\Theta}$, where $\boldsymbol{\Theta}$ is any off-diagonal Hermitian matrix such that $\mathbf{C}_{\alpha}+\mathbf{\Theta} \succeq \mathbf{0}$. Notice that, if $R_{\mathrm{PPL}}(\mathbf{Q}) \geq R_{\mathrm{PPL}}\left(\mathbf{C}_{\alpha}\right)$ holds for all $\Theta$, then $R_{\mathrm{PPL}}\left(\mathbf{Q}^{\prime}\right) \geq R_{\mathrm{PPL}}\left(\mathbf{C}_{\alpha}\right)$ for any $\overline{\mathbf{Q}}^{\prime} \preceq_{\mathcal{D}} \mathbf{Q}$. The lemma is therefore proved if the following holds

$$
\operatorname{det}\left(\mathbf{I}+\left(\overline{\mathbf{C}}_{\alpha}+\boldsymbol{\Theta}\right)^{-1} \boldsymbol{\Sigma}_{S}\right) \geq \operatorname{det}\left(\mathbf{I}+\overline{\mathbf{C}}_{\alpha}^{-1} \boldsymbol{\Sigma}_{S}\right) .
$$

Applying the determinant identities $\operatorname{det}(\mathbf{A B})=\operatorname{det}(\mathbf{A}) \operatorname{det}(\mathbf{B})$, for any squared matrices $\mathbf{A}$ and $\mathbf{B}$, and $\operatorname{det}\left(\mathbf{A}^{-1}\right)=1 / \operatorname{det}(\mathbf{A})$; the foregoing expression can be equivalently given by

$$
\frac{\operatorname{det}\left(\overline{\mathbf{C}}_{\alpha}+\boldsymbol{\Sigma}_{S}+\boldsymbol{\Theta}\right)}{\operatorname{det}\left(\overline{\mathbf{C}}_{\alpha}+\boldsymbol{\Sigma}_{S}\right)} \geq \frac{\operatorname{det}\left(\overline{\mathbf{C}}_{\alpha}+\boldsymbol{\Theta}\right)}{\operatorname{det}\left(\overline{\mathbf{C}}_{\alpha}\right)} .
$$

As $\operatorname{det}(\mathbf{A}) \leq \prod_{i}(\mathbf{A})_{i i}$, with equality only when $\mathbf{A}$ is diagonal, and $\boldsymbol{\Sigma}_{S}$ is a diagonal matrix with positive entries, (10) holds for any $\boldsymbol{\Theta}$, which concludes the proof. 


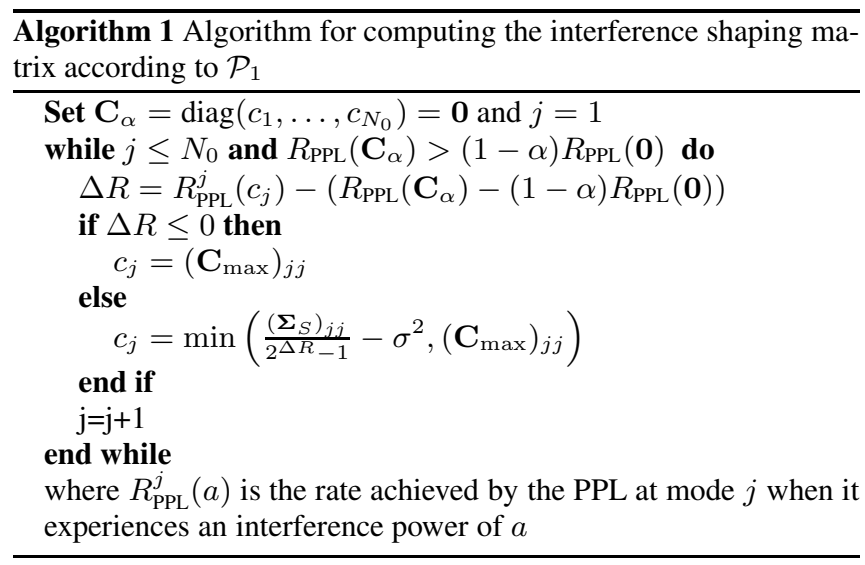

Note that the ordering (5) cannot be used for the transmit covariance constraints since the diagonal of $\mathbf{Q}$ depends on the off-diagonal elements of the transmit covariances. Finally, using Lemma 1, it is easy to see that the transmit covariances satisfying (8) also satisfy (6) for all $\mathbf{S}_{j}$ such that $\sum_{j=1}^{K} \mathbf{U}_{0}^{H} \mathbf{H}_{0 j} \mathbf{S}_{j} \mathbf{H}_{0 j}^{H} \mathbf{U}_{0} \preceq_{\mathcal{D}} \mathbf{C}_{\alpha}$.

\section{ALGORITHM DESIGN}

In this section we propose algorithms for computing $\mathbf{C}_{\alpha}$ and $\mathbf{S}_{j}$, $j=1, \ldots, K$, as well as an extension of the MinIL algorithm that incorporates the transmit covariance constraints (8) and a power control step.

\subsection{Shaping constraint design}

The interference shaping constraint, $\mathbf{C}_{\alpha}$, must be chosen such that $R_{\mathrm{PPL}}\left(\mathbf{C}_{\alpha}\right)=(1-\alpha) R_{\mathrm{PPL}}(\mathbf{0})$ and it is the least stringent for the IC. To this end, we propose the following optimization problem

$$
\begin{array}{lll}
\mathcal{P}_{1}: & \underset{\mathbf{C}_{\alpha}}{\operatorname{maximize}} & \operatorname{Tr}\left(\mathbf{C}_{\alpha}\right), \\
\text { subject to } & R_{\mathrm{PPL}}\left(\mathbf{C}_{\alpha}\right) \geq(1-\alpha) R_{\mathrm{PPL}}(\mathbf{0}), \\
& \mathbf{0} \preceq \mathbf{C}_{\alpha} \preceq \mathbf{C}_{\max },
\end{array}
$$

where $\left(\mathbf{C}_{\max }\right)_{i i}=\left(\sum_{j=1}^{K} \mathbf{U}_{0}^{H} \mathbf{H}_{0 j} \mathbf{H}_{0 j}^{H} \mathbf{U}_{0}\right)_{i i}$ and zeros elsewhere. Recall that, according to (7), the optimal $\mathbf{C}_{\alpha}$ is a diagonal matrix. In $\mathcal{P}_{1}$, the allowed interference power at the PPL is maximized subject to the minimum rate constraint and an additional constraint that bounds the maximum allowed interference level at each stream to the worst case, which is represented by each entry of $\mathbf{C}_{\max }$. This may occur if the transmit directions are aligned to the channels from the transmitters to the PPL receiver. To solve this non-convex problem, we use the ensuing lemma.

Lemma 3. Let us denote by $\mathbf{C}_{\alpha}^{\star}=\operatorname{diag}\left(c_{1}^{\star}, \ldots, c_{N_{0}}^{\star}\right)$ the optimal solution of $\mathcal{P}_{1}$, where $c_{j}^{\star}$ is associated to the $j$ th weakest mode of the PPL channel. Then, the following holds

$$
c_{j}^{\star}<\left(\mathbf{C}_{\max }\right)_{j j} \Rightarrow c_{j+1}^{\star}=0, j=1, \ldots, N_{0}-1 .
$$

Proof. As $\mathbf{C}_{\alpha}$ is diagonal, we have $R_{\mathrm{PPL}}\left(\mathbf{C}_{\alpha}\right)=\sum_{j} \log [1+$ $\left.\left(\boldsymbol{\Sigma}_{S}\right)_{j j} /\left(\sigma^{2}+c_{j}\right)\right]$. The derivative of $R_{\mathrm{PPL}}\left(\mathbf{C}_{\alpha}\right)$ with respect to $c_{j}, \nabla_{c_{j}} R_{\mathrm{PPL}}$, is monotone decreasing and $\nabla_{c_{j}} R_{\mathrm{PPL}} \leq \nabla_{c_{j+1}} R_{\mathrm{PPL}}$ for $c_{j}=c_{j+1}, j=1, \ldots, N_{0}-1$, i.e., the weaker the mode, the more interference power it tolerates to meet a given data rate. As the interference level is limited by $\mathbf{C}_{\max }$, we obtain (11), which concludes the proof.
The above lemma allows us to find the optimal solution of $\mathcal{P}_{1}$ stream-wise, as detailed in Algorithm 1. Similarly, using the solution of $\mathcal{P}_{1}$, we propose the following convex optimization problem for computing the transmit covariance constraints

$$
\begin{aligned}
& \mathcal{P}_{2}: \operatorname{maximize}_{\left\{\mathbf{S}_{j}\right\}_{1}^{K}}^{\max } \sum_{j=1}^{K} \operatorname{Tr}\left(\mathbf{S}_{j}\right), \\
& \text { subject to } \\
& \sum_{j=1}^{K} \mathbf{U}_{0}^{H} \mathbf{H}_{0 j} \mathbf{S}_{j} \mathbf{H}_{0 j}^{H} \mathbf{U}_{0} \preceq_{\mathcal{D}} \mathbf{C}_{\alpha}, \\
& \mathbf{0} \preceq \mathbf{S}_{j} \preceq \mathbf{I}, j=1, \ldots, K,
\end{aligned}
$$

where the last constraint is due to the unitary transmit power of the secondary transmitters. Recall that $\mathcal{P}_{1}$ and $\mathcal{P}_{2}$ are both solved at the primary user. The obtained transmit covariance constraints, $\mathbf{S}_{j}$, are then sent to the IC, which performs the MinIL algorithm as described in the next subsections. Notice that the PPL could modify its transmission strategy to exploit the knowledge about the interference shaping constraint, $\mathbf{C}_{\alpha}$, which would result in a coupled problem. For example, a worst-case optimization at the PPL would assume that the aggregate interference covariance matrix is equal to $\mathbf{C}_{\alpha}$, what would change the optimal power allocation at the PPL, but not the transmit directions. For simplicity, we consider in this paper that the PPL does not change its transmission strategy and we leave further PPL optimization for future work.

\subsection{Extension of MinIL algorithm}

At each step of the MinIL, the precoders (decoders) are optimize subject to norm constraints, while the decoders (precoders) are fixed, so that the IL is successively minimized [10]. Notice that in our model, the additional constraint affects only the design of the precoders, whereas the decoders are optimized exactly as in the original algorithm. The IL is given by

$$
i_{\text {leak }}=\sum_{i=1}^{K} \sum_{j \neq i}^{K}\left|\mathbf{u}_{i}^{H} \mathbf{H}_{i j} \mathbf{v}_{j}\right|^{2} .
$$

Therefore, for fixed precoders, the unit-norm decoders that minimize (12) are given by $\mathbf{u}_{i}^{\star}=\nu_{\min }\left(\sum_{j \neq i}^{K} \mathbf{H}_{i j} \mathbf{v}_{j} \mathbf{v}_{j}^{H} \mathbf{H}_{i j}^{H}\right)$, where $\nu_{\min }(\mathbf{A})$ denotes the eigenvector of $\mathbf{A}$ with minimum eigenvalue. On the other hand, when the decoders are kept fixed, the optimal precoder of the $j$ th transmitter is obtained by solving the following optimization problem

$$
\begin{array}{lll}
\mathcal{P}_{3}: & \underset{\mathbf{v}_{j}}{\operatorname{minimize}} & i_{\text {leak }}, \\
\text { subject to } & \mathbf{v}_{j}^{H} \mathbf{v}_{j}=\min \left(1, \operatorname{Tr}\left(\mathbf{S}_{j}\right)\right), \\
& \mathbf{v}_{j} \mathbf{v}_{j}^{H} \preceq \mathbf{S}_{j} .
\end{array}
$$

To solve the foregoing problem, we first rewrite the transmit covariance constraint by using the following lemma.

Lemma 4. Let $\mathbf{S} \in \mathcal{S}_{+}^{N}$ and $\mathbf{v} \in \mathcal{C}^{N \times 1}$. Then $\mathbf{v v}^{H} \preceq \mathbf{S}$ holds if and only if $\left\|\mathbf{\Sigma}^{-\frac{1}{2}} \mathbf{F}^{H} \mathbf{v}\right\|^{2} \leq 1$, where $\mathbf{S}=\mathbf{F} \mathbf{\Sigma} \mathbf{F}^{H}$ is the singular value decomposition of $\mathbf{S}$.

Proof. Taking the singular value decomposition of $\mathbf{S}, \mathbf{v} \mathbf{v}^{H} \preceq \mathbf{S}$ is equivalent to $\boldsymbol{\Sigma}^{-\frac{1}{2}} \mathbf{F}^{H} \mathbf{v} \mathbf{v}^{H} \mathbf{F} \boldsymbol{\Sigma}^{-\frac{1}{2}} \preceq \mathbf{I}$. Therefore, the maximum eigenvalue of $\boldsymbol{\Sigma}^{-\frac{1}{2}} \mathbf{F}^{H} \mathbf{v} \mathbf{v}^{H} \mathbf{F} \boldsymbol{\Sigma}^{-\frac{1}{2}}$ must be equal or lower than 1 . Since this matrix is rank-one, its maximum eigenvalue is given by $\left\|\boldsymbol{\Sigma}^{-\frac{1}{2}} \mathbf{F}^{H} \mathbf{v}\right\|^{2}$, which concludes the proof. 


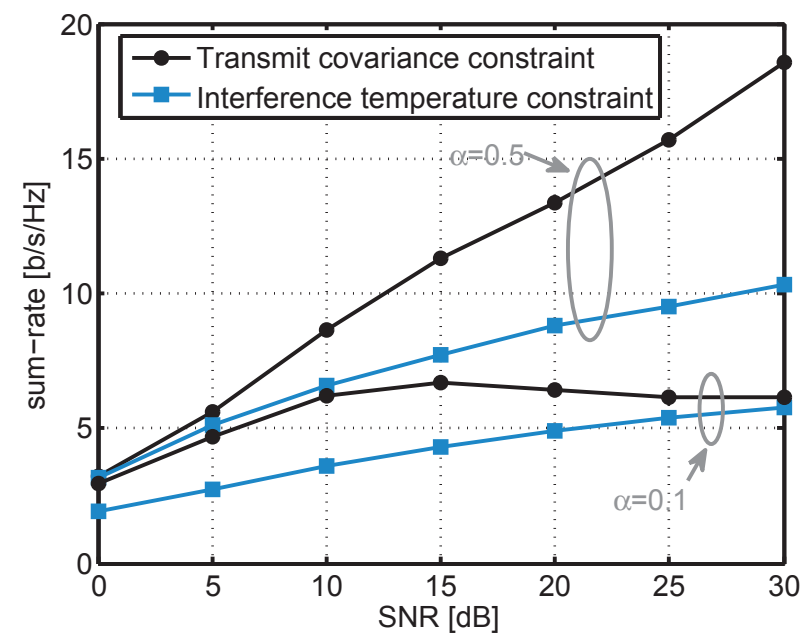

Fig. 1. Achievable sum-rate of the $(3 \times 2,1)^{3}$ IC with a $3 \times 3$ PPL for both interference constraints and different values of $\alpha$. obtain

Using the foregoing lemma and taking the Lagrangian of $\mathcal{P}_{3}$, we

$$
\mathbf{v}_{j}^{\star}=\sqrt{\min \left(1, \operatorname{Tr}\left(\mathbf{S}_{j}\right)\right)} \nu_{\min }\left[\left(1-\mu_{j}\right) \mathbf{R}_{j}+\mu_{j} \mathbf{S}_{j}^{-1}\right],
$$

where $\mathbf{R}_{j}=\sum_{i \neq j}^{K} \mathbf{H}_{i j}^{H} \mathbf{u}_{i} \mathbf{u}_{i}^{H} \mathbf{H}_{i j}$ and $\mu_{j} \in[0,1]$, which can be obtained using bisection, such that the equivalent transmit covariance constraint is satisfied with equality (if active). We omit the details due to lack of space.

\subsection{Power control}

Even when the IA problem is feasible, the IC may not achieve zero IL due to the additional shaping constraint, specially for low values of $\alpha$. In these cases it is important to control the power transmitted by the IC to improve its sum-rate. However, if we relax the power constraint in $\mathcal{P}_{3}$ by an inequality, we will obtain zero transmit power. Since we do not want to reduce the IL by means solely of reducing the transmit power, a normalizing term must be included in $\mathcal{P}_{3}$. To this end, when the IL converges to a non-zero value, each transmitter computes a new precoder by solving the following problem

$$
\begin{array}{cl}
\mathcal{P}_{4}: \quad \underset{\tilde{\mathbf{v}}_{j}, p_{j}}{\operatorname{minimize}} & p_{j}\left[i_{\text {leak }}\left(\tilde{\mathbf{v}}_{j}\right)-i_{\text {leak }}\left(\mathbf{v}_{j}^{\prime}\right)\right], \\
\text { subject to } & \tilde{\mathbf{v}}_{j}^{H} \tilde{\mathbf{v}}_{j}=\min \left(1, \operatorname{Tr}\left(\mathbf{S}_{j}\right)\right), \\
& p_{j} \tilde{\mathbf{v}}_{j} \tilde{\mathbf{v}}_{j}^{H} \preceq \mathbf{S}_{j}, \\
& p_{j} \leq 1,
\end{array}
$$

where $i_{\text {leak }}\left(\mathbf{v}_{j}\right)$ is the IL when the $j$ th transmitter applies $\mathbf{v}_{j}$, and $\mathbf{v}_{j}^{\prime}$ is the precoder from the previous iteration $\left(i_{\text {leak }}\left(\mathbf{v}_{j}^{\prime}\right)\right.$ is therefore the current IL). The new precoder is then given by $\mathbf{v}_{j}^{\star}=\sqrt{p_{j}^{\star}} \tilde{\mathbf{v}}_{j}^{\star}$, i.e., $p_{j}$ is the ratio between the transmit power and the power budget of user $j$. In the objective function of $\mathcal{P}_{4}$, the effect on the IL of the transmit direction and the transmit power is decoupled by $\left[i_{\text {leak }}\left(\tilde{\mathbf{v}}_{j}\right)-i_{\text {leak }}\left(\mathbf{v}_{j}^{\prime}\right)\right]$ and $p_{j}$, respectively. The optimization of the transmit power by means of $p_{j}$ allows to explore other transmit directions which may reduce $\left[i_{\text {leak }}\left(\tilde{\mathbf{v}}_{j}\right)-i_{\text {leak }}\left(\mathbf{v}_{j}^{\prime}\right)\right]$. However, the optimal value of $\mathcal{P}_{4}$ is equal or lower than zero, and thus reducing the transmit power may not reduce the objective function, which is hence minimized when a compromise between the transmit direction

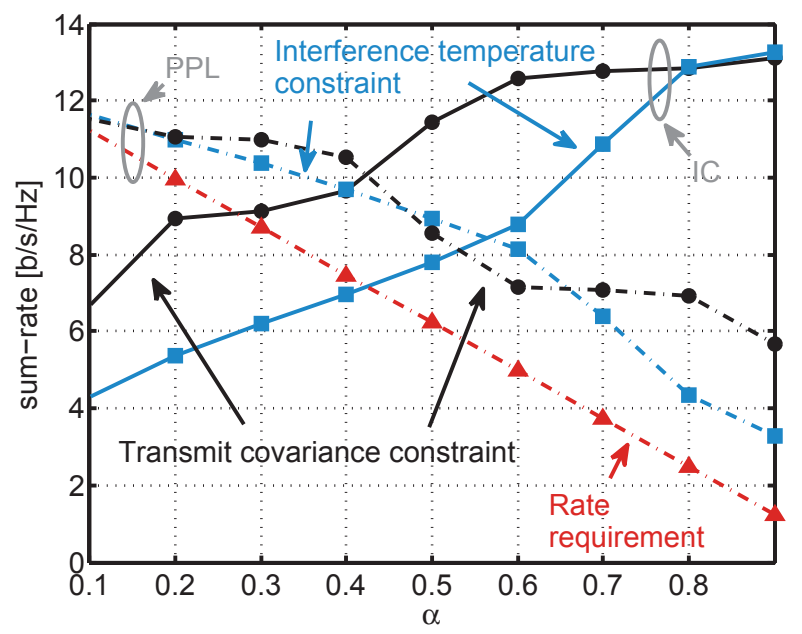

Fig. 2. Achievable sum-rate of the $(3 \times 2,1)^{3}$ IC and the $3 \times 3$ PPL as a function of $\alpha$ for $\mathrm{SNR}=15 \mathrm{~dB}$.

and $p_{j}$ is reached. The new precoder, $\mathbf{v}_{j}^{\star}$, is the scaled generalized eigenvector with minimum generalized eigenvalue of the matrix pencil $\left(\mathbf{R}_{j}-\frac{\mathbf{v}_{j}^{\prime H} \mathbf{R}_{j} \mathbf{v}_{j}^{\prime}}{\min \left(1, \operatorname{Tr}\left(\mathbf{S}_{j}\right)\right)} \mathbf{I}, \mathbf{S}_{j}^{-1}\right)$. We omit the details due to lack of space.

\section{NUMERICAL RESULTS}

We consider a $(3 \times 2,1)^{3}$ IC and a $3 \times 3$ PPL, and define SNR $=$ $10 \log _{10}\left(1 / \sigma^{2}\right)$. The entries of the channel matrices are i.i.d. complex Gaussian random variables with zero mean and unit variance. All results are averaged over 1000 different channel realizations. For the IT constraint we use the algorithm proposed in [22] with the additional power control step (by solving an analogous problem to $\mathcal{P}_{4}$ ) and choosing the IT constraint such that the rate requirement is guaranteed. In Fig. 1 we show the achievable sum-rate of the IC for different values of $\alpha$ and for both interference constraints. Whereas with both constraints the rate of the PPL is guaranteed, the transmit covariance constraint allow the IC to achieve much higher data rates than the IT thanks to controlling the spatial interference distribution at the PPL. Moreover, the transmit covariance constraint allows the IC to optimize its precoders and decoders without actually knowing the channel to the PPL. Alternatively, we depict in Fig. 2 the sumrate of the IC at SNR $=15 \mathrm{~dB}$ as a function of $\alpha$, where it can be observed the high performance improvement of the transmit covariance constraint over most of the range of $\alpha$, showing again that controlling the spatial structure of the interference plays an important role when the PPL transmits multiple streams.

\section{CONCLUSION}

In this paper we have studied network coexistence between an IC and a PPL in the context of CR. We have shown that controlling the spatial structure of the interference is critical in order to provide high sum-rate to the IC, while ensuring the rate requirement at the PPL. We have then extended the MinIL algorithm to incorporate such constraints and an additional power control step to enhance the sum-rate of the IC. We have shown through different numerical examples the importance of controlling the spatial structure of the interference when the PPL transmits multiple streams. 


\section{REFERENCES}

[1] S. Haykin, "Cognitive radio: brain-empowered wireless communications," IEEE J. Sel. Areas Commun., vol. 23, no. 2, pp. 201-220, Feb. 2005.

[2] B. A. Fette, Cognitive Radio Technology. Newnes, 2006.

[3] V. Cadambe and S. Jafar, "Interference alignment and degrees of freedom of the k -user interference channel," IEEE Trans. Inf. Theory, vol. 54, no. 8, pp. 3425-3441, Aug. 2008.

[4] M. Maddah-Ali, A. Motahari, and A. Khandani, "Communication over MIMO X channels: Interference alignment, decomposition, and performance analysis," IEEE Trans. Inf. Theory, vol. 54, no. 8, pp. 3457-3470, Aug. 2008.

[5] S. A. Jafar, "Interference alignment: A new look at signal dimensions in a communication network," Foundations and Trends Commun. Inf. Theory, vol. 7, no. 1, pp. 1-136, 2011.

[6] C. Wang, T. Gou, and S. Jafar, "Subspace alignment chains and the degrees of freedom of the three-user mimo interference channel," in Proc. IEEE Int. Symp. Information Theory (ISIT), Jul. 2012, pp. 2471-2475.

[7] S. A. Jafar and M. Fakhereddin, "Degrees of freedom for MIMO interference channel," IEEE Trans. Inf. Theory, vol. 53, no. 7, pp. 2637-2642, Jul. 2007.

[8] Ó. González, I. Santamaría, and C. Beltrán, "A general test to check the feasibility of linear interference alignment," in Proc. of the IEEE International Symposium on Information Theory (ISIT), Jul. 2012, pp. 2481-2485.

[9] Ó. González, C. Beltrán, and I. Santamaría, "A feasibility test for linear interference alignment in MIMO channels with constant coefficients," IEEE Trans. Inf. Theory, In Press.

[10] K. Gomadam, V. Cadambe, and S. Jafar, "A distributed numerical approach to interference alignment and applications to wireless interference networks," IEEE Trans. Inf. Theory, vol. 57, no. 6, pp. 3309-3322, Jun. 2011.

[11] S. Peters and R. Heath, "Interference alignment via alternating minimization," in Proc. IEEE Int. Conf. Acoustics, Speech and Signal Processing, (ICASSP), Apr. 2009, pp. 2445-2448.

[12] H. Yu and Y. Sung, "Least squares approach to joint beam design for interference alignment in multiuser multi-input multioutput interference channels," IEEE Trans. Signal Process., vol. 58, no. 9, pp. 4960-4966, Sep. 2010.

[13] R. W. Heath Jr. and S. Peters, "Cooperative algorithms for MIMO interference channels," IEEE Trans. Veh.Technol., vol. 60, no. 1, pp. 206-218, Jan. 2011.

[14] D. Schmidt, C. Shi, R. Berry, M. L. Honig, and W. Utschick, "Minimum mean squared error interference alignment," in Proc. Asilomar Conf. Signals, Systems and Computers, Nov. 2009, pp. 1106-1110.

[15] S.-H. Park, H. Park, Y.-D. Kim, and I. Lee, "Regularized interference alignment based on weighted sum-MSE criterion for MIMO interference channels," in Proc. IEEE Int. Conf. Communications (ICC), May 2010, pp. 1-5.

[16] I. Santamaría, Ó. González, R. W. Heath Jr., and S. W. Peters, "Maximum sum-rate interference alignment algorithms for MIMO channels," in Proc. IEEE Global Communications Conf. (GLOBECOM), Dec. 2010, pp. 1-6.
[17] C. Lameiro, Ó. González, and I. Santamaría, "An interference alignment algorithm for structured channels," in Proc. IEEE Work. Signal Processing Advances in Wireless Communications (SPAWC), Jun. 2013, pp. 295-299.

[18] M. Amir, A. El-Keyi, and M. Nafie, "Constrained interference alignment and the spatial degrees of freedom of MIMO cognitive networks," IEEE Trans. Inf.Theory, vol. 57, no. 5, pp. 2994-3004, May 2011.

[19] G. Chen, Z. Xiang, C. Xu, and M. Tao, "On degrees of freedom of cognitive networks with user cooperation," IEEE Wireless Commun. Lett., vol. 1, no. 6, pp. 617-620, Dec. 2012.

[20] B. Koo and D. Park, "Interference alignment with cooperative primary receiver in cognitive networks," IEEE Commun. Lett., vol. 16, no. 7, pp. 1072-1075, Jul. 2012.

[21] B. Abdelhamid, M. ElSabrouty, and S. Elramly, "Novel interference alignment in multi-secondary users cognitive radio system," in Proc. IEEE Symp. Computers and Communications (ISCC), Jul. 2012, pp. 785-789.

[22] H. Du, T. Ratnarajah, H. Zhou, and Y.-C. Liang, "Interference alignment for peer-to-peer underlay MIMO cognitive radio network," in Proc. IEEE Asilomar Conf. Signals, Systems and Computers, Nov. 2011, pp. 349-353.

[23] R. Zhang and Y.-C. Liang, "Exploiting multi-antennas for opportunistic spectrum sharing in cognitive radio networks," IEEE J. Sel. Topics Signal Process., vol. 2, no. 1, pp. 88-102, Feb. 2008.

[24] R. Zhang, "Optimal power control over fading cognitive radio channel by exploiting primary user CSI," in Proc. IEEE Global Telecommunications Conf. (GLOBECOM), Nov. 2008, pp. 15

[25] K. Cumanan, R. Zhang, and S. Lambotharan, "A new design paradigm for MIMO cognitive radio with primary user rate constraint," IEEE Commun. Lett., vol. 16, no. 5, pp. 706-709, May 2012.

[26] J. Lv and E. Jorswieck, "Spatial shaping in cognitive system with coded legacy transmission," in Proc. Int. ITG Work. Smart Antennas (WSA), Feb. 2011, pp. 1-6.

[27] E. Jorswieck and J. Lv, "Spatial shaping in cognitive MIMO MAC with coded legacy transmission," in Proc. IEEE Int. Work. Signal Processing Advances in Wireless Communications (SPAWC), Jun. 2011, pp. 451-455.

[28] A. Dotzler, M. Riemensberger, W. Utschick, and G. Dietl, "Interference robustness for cellular MIMO networks," in Proc. IEEE Int. Work. Signal Processing Advances in Wireless Communications (SPAWC), Jun. 2012, pp. 229-233.

[29] I. E. Telatar, "Capacity of multi-antenna gaussian channels," European Trans. Telecommun., vol. 10, no. 6, pp. 585-595, Nov. 1999.

[30] A. W. Marshall, I. Olkin, and B. C. Arnold, Inequalities : theory of majorization and its applications, ser. Springer series in statistics. New York: Springer, 2011.

[31] D. A. Simovici and C. Djeraba, Mathematical tools for data mining : set theory, partial orders, combinatorics, ser. Advanced information and knowledge processing. London: Springer, 2008. 